\title{
Sextant
}

Revue de recherche interdisciplinaire sur le genre et la sexualité

36 | 2019

No children, no cry

\section{Revendiquer la non-maternité pour ré-engen(d)rer la mémoire chez trois écrivaines de langue française}

Nathalie Ségeral

\section{(2) OpenEdition}

1 Journals

Édition électronique

URL : https://journals.openedition.org/sextant/393

DOI : $10.4000 /$ sextant.393

ISSN : 2795-8736

Éditeur

Éditions de l'Université de Bruxelles

Édition imprimée

Date de publication : 1 décembre 2019

Pagination : 135-151

ISBN : 978-2-8004-1705-9

ISSN : 1370-267X

Référence électronique

Nathalie Ségeral, « Revendiquer la non-maternité pour ré-engen(d)rer la mémoire chez trois écrivaines de langue française », Sextant [En ligne], 36 | 2019, mis en ligne le 01 novembre 2021, consulté le 08 décembre 2021. URL : http://journals.openedition.org/sextant/393 ; DOI : https://doi.org/10.4000/ sextant.393

\section{(ब) $\odot$}

La revue Sextant est mise à disposition selon les termes de la Licence Creative Commons Attribution Pas d'Utilisation Commerciale - Partage dans les Mêmes Conditions 4.0 International. 


\title{
Revendiquer la non-maternité pour ré-engen(d)rer la mémoire chez trois écrivaines de langue française
}

\author{
Nathalie SÉGERAL
}

\section{Résumé :}

Tandis que la plupart des récits de survivantes de catastrophes historiques mettent l'accent sur la maternité comme revanche biologique et catharsis, cette étude se concentre sur trois textes revendiquant la non-maternité volontaire dans un contexte mémoriel traumatique. A l'enfant que je n'aurai pas (2011), de Linda Lê, est un récit autobiographique épistolaire adressé à l'enfant imaginé de l'auteure-narratrice, dans lequel elle évoque les raisons de son choix de ne pas se reproduire dans le contexte du double traumatisme d'une mère toxique et de l'exil ; Maternité (2018), de Françoise Guérin, raconte la trajectoire parallèle de deux sæurs ayant souffert de violence psychologique de la part de leur mère, l'une choisissant de ne pas avoir d'enfant biologique et d'adopter, espérant ainsi briser le cycle mortifère, l'autre sombrant dans la dépression post-natale ; L'Amour après (2018), de Marceline Loridan-Ivens, survivante de la Shoah, expose la non-maternité choisie de l'auteure-narratrice comme condition de libération de la prison psychologique du traumatisme de l'expérience concentrationnaire, parallèlement à son engagement dans le Mouvement de libération des femmes. Très peu d'études ont jusqu'ici été consacrées à la nonmaternité choisie et aucune ne s'est encore penchée sur la non-maternité volontaire dans un contexte traumatique complexe. Ces trois textes font écho à la métaphore du " corps silencieux " décrite par Peterson et Engwall, permettant une essentialisation du choix de ne pas procréer en évoquant l'absence d'instinct maternel. Ceci permet à ces femmes de se positionner au sein de la dichotomie mère/non-mère sans avoir à la rationaliser, grâce au recours à une autre forme de déterminisme biologique.

\begin{abstract}
:
While most female genocide survivors' testimonies highlight motherhood as cathartic and as a biological revenge, this study focuses on three narratives reclaiming voluntary childlessness in the wake of trauma. Linda Lê's A l'enfant que je n'aurai pas [To the Child I Will Not Have] (2018) is an autobiographical text in which the narrator-author details her reasons for remaining childfree in the traumatic context of a toxic mother and exile. Françoise Guérin's Maternité [Motherhood] (2018) tells the parallel stories of two sisters who experienced psychological abuse by their mother. One of them chooses not to have biological children and to adopt, hoping to break the destructive cycle of trauma, while the other one suffers from severe postpartum depression. Holocaust survivor Marceline Loridan-Ivens's L'Amour après [Love After] (2018) presents the author-narrator's voluntary childlessness as a means to
\end{abstract}


her liberation from the mental prison of the concentrationary universe, paralleling her struggle for women's liberation. Very few studies have so far been devoted to voluntary childlessness and none has yet explored voluntary childlessness in the wake of trauma. These three texts echo the "silent body" metaphor described by Peterson and Engwall, allowing these women to essentialize the choice not to reproduce by talking about the lack of maternal instinct. Thus, these women position themselves within the mother/non-mother dichotomy without having to rationalize their choice, by resorting to another form of biological determinism.

\section{Introduction}

La plupart des récits de survivantes de génocides, notamment de la Shoah et du génocide des Tutsis du Rwanda, mettent l'accent sur l'accès à la maternité comme faisant partie intégrante du processus cathartique, comme si se marier et perpétuer la généalogie interrompue par l'irruption de la catastrophe historique permettait de conjurer le sort et constituait une forme de revanche biologique. Or, les écrivaines incluses dans cette étude vont à rebours de cette tradition en revendiquant la nonmaternité choisie dans un contexte de traumatisme historique et personnel. Au cours des cinq dernières années, quelques articles scientifiques et études ont commencé à s'attacher à la non-maternité volontaire, dans ses causes et sa construction narrative (Letherby, Peterson et Engwall), ainsi qu'à l'expérience genrée du génocide dans le désir de maternité des survivantes (Waxman). Cependant, aucune étude ne s'est encore à ce jour penchée sur la non-maternité volontaire dans un contexte mémoriel traumatique complexe. Ainsi, comment et pourquoi survivre ou revivre en choisissant de ne pas donner la vie?

Dans son ultime ouvrage, L'Amour après ${ }^{1}$, paru peu avant son décès à l'automne 2018, la survivante de la Shoah Marceline Loridan-Ivens prend pour point de départ la relecture de lettres reçues au cours de sa vie pour évoquer un sujet encore tabou : celui du rapport au corps et à la sexualité dans le sillage de la Shoah et, notamment, sa décision de ne pas avoir d'enfants, contrairement à la majorité de ses camarades ayant survécu - dont Simone Veil, avec laquelle elle a partagé sa captivité à Auschwitz. L'auteure franco-vietnamienne Linda Lê publie $A$ l'enfant que je n'aurai pas ${ }^{2}$ (2011) dans la collection «Les affranchies » des éditions Nil, une série consacrée aux auteur·e·s désirant rédiger une lettre qu'ils n'ont jamais auparavant osé écrire. Lê choisit donc d'adresser la sienne à « toi », l'enfant biologique qu'elle n'a pas eu et qu'elle ne pourra plus avoir. Enfin, Françoise Guérin, dans son roman Maternité (2018), s’inspire de son expérience clinique de psychothérapeute spécialisée dans le lien mère-enfant pour livrer au lecteur un récit dérangeant sur la confrontation d'une jeune mère à l'absence totale d'instinct maternel et sa lente descente dans l'enfer de la dépression du post-partum, parallèlement à l'histoire de sa sœur qui, évoquée en

\footnotetext{
1 M. Loridan-Ivens, L'Amour après, Paris, Grasset, 2018.

${ }^{2}$ L. LÊ, A l'Enfant que je n'aurai pas, Paris, NiL, 2011.
} 
filigrane, a choisi de ne pas se reproduire afin d'échapper à la malédiction familiale liée à la maltraitance maternelle dont elles ont toutes deux souffert.

Très peu d'études ont donc jusqu'ici été consacrées à la non-maternité volontaire. Helen Peterson et Kristina Engwall, dans leur étude de la non-maternité volontaire chez une trentaine de Suédoises âgées de 29 ans à 54 ans, intitulée « Silent Bodies: Childfree women's gendered and embodied experience », évoquent la métaphore du « corps silencieux » ( « silent body » ${ }^{3}$ ) pour résumer les arguments donnés par la plupart de ces femmes afin d'expliquer leur « childfreeness » (le terme anglais ayant une connotation beaucoup plus positive que le français « sans enfant»). En effet, cette incarnation et essentialisation de leur choix de ne pas procréer, en évoquant l'absence fondamentale d'instinct maternel ou de sentiment d'horloge biologique, leur permet de se positionner au sein de la dichotomie mère / non-mère sans avoir à la rationaliser, grâce au recours à une autre forme de déterminisme biologique faisant écho à celui utilisé par la société pour inciter les femmes à se reproduire. « Être mère, t'a-t-on répété, c'est tellement naturel... Ne devrais-tu pas te fier à ton instinct ? ${ }^{4}$, écrit Guérin, se faisant l'écho de l'injonction sociale face à l'angoisse de la jeune mère ne ressentant aucun attachement pour son nouveau-né.

Linda Lê et Marceline Loridan-Ivens s'inscrivent dans la lignée d'autres ouvrages récents revendiquant la non-maternité, tels L'Hydre de Lerne ${ }^{5}$ de Cécile Wajsbrot (2011), Je dois tout à ton oubli de Malika Mokeddem (2008) et Nullipare ${ }^{6}$ de Jane Sautière (2008), et font, à ce titre, partie de ce qui pourrait être considéré comme une tendance émergeante au sein de la littérature française et francophone contemporaine : la réappropriation du désir de demeurer sans enfant et le besoin de rédiger un texte aux allures de manifeste à ce sujet, dans un contexte mémoriel compliqué. Ces ouvrages contribuent à une redéfinition de la notion de « maternité » en remettant en question ses principes fondateurs et en utilisant la littérature comme moyen privilégié pour exposer le choix de ces narratrices de ne pas se reproduire, dans le contexte d'une société - la France - qui se targue actuellement d'un des taux de natalité les plus élevés d'Europe et dont la politique sociale et familiale souligne lourdement la maternité comme but et accomplissement ultimes.

Lê, Loridan-Ivens et Guérin contribuent ainsi à la redéfinition du concept de « mère " en en questionnant les fondements et les motivations. Cette revendication est, dans les trois cas, compliquée par la problématique de la mémoire et, parfois (dans le cas de Guérin notamment), de la post-mémoire. Ainsi, selon Marianne Hirsch, la post-mémoire - concept développé dans le contexte de la Shoah mais pouvant être étendu à tout autre traumatisme transgénérationnel - consiste en la transmission intergénérationnelle d'un traumatisme et passe, le plus souvent, par la relation mèrefille, censée être plus fusionnelle et plus intense émotionnellement que celle entre mère et fils - concept certes discutable. Or, chez ces trois auteures, le refus de la maternité s'accompagne aussi d'un rejet de la figure maternelle, qui s'apparente, au

${ }^{3}$ H. Peterson et Kr. Engwall, « Silent Bodies: Childfree Women's Gendered and Embodied Experiences », European Journal of Women's Studies, 20, 2013, p. 379.

${ }^{4}$ Fr. GUÉRIN, op. cit., p. 149.

${ }^{5}$ C. Wajsbrot, L'Hydre de Lerne, Paris, Denoël, 2011.

${ }^{6}$ J. SAUtiÈre, Nullipare, Paris, Gallimard, 2008. 
fil du texte, à un rejet du poids de la mémoire, des origines, et d'un passé qui ne passe pas, d'où un refus de la maternité présenté comme refus de transmission. Nous étudierons donc les procédés littéraires utilisés à cette fin de (ré)enfantement de la mémoire par la revendication de la non-maternité.

En effet, allant plus loin que l'étude de Natalie Edwards intitulée Voluntary Childlessness $^{7}$, tout en la nuançant, cet essai émet l'argument selon lequel, bien que ces récits puissent se lire comme des manifestes pour la non-maternité, ils se trouvent fortement compliqués par des problématiques liées à la mémoire. Le refus de la maternité est étroitement lié à un rejet de la figure maternelle, voire de la langue maternelle, ce qui s'apparente finalement à un rejet du poids de la mémoire, des origines et du traumatisme qui enferme (qu'il s'agisse de la Shoah, de la Guerre du Viêtnam, ou de l'exil et de l'immigration), d'où un rejet de la maternité présenté avant tout comme refus de transmettre, l'enfant se réduisant à l'incarnation vivante d'un mémorial et n'étant plus perçu comme ouverture sur l'avenir.

Lê remet également en question la notion de déracinement au cœur des récits d'exil et d'immigration, développée récemment dans le contexte des études féministes américaines par Marianne Hirsch et Nancy Miller dans Rites of Return: Diaspora Poetics and the Politics of Memory ${ }^{8}$. Au contraire, son récit autobiographique sur le choix de demeurer sans enfant souligne le rejet d'un certain discours des origines et, de ce fait, le rejet du mythe de la mélancolie envers des racines romancées et idéalisées. «L'origine » (le Viêtnam, en l'occurrence) est souvent violemment rejetée, ce qui conduit à un rejet de la figure maternelle en tant que véhicule et symbole de cette origine méprisée. Dans un entretien de 2010 pour Télérama, Lê déclarait :

Je me sens presque toujours en exil [dans la France d'aujourd'hui]. Je crois que même si je vis en France depuis longtemps je ne me suis jamais dit : là est mon pays. Mais je ne me dis pas non plus que le Viêtnam est mon pays. Je porte à la langue française un amour profond. C'est mon seul point d'ancrage dans une réalité que je continue de trouver très violente ${ }^{9}$.

Cette étude se propose donc d'explorer plusieurs facettes de ce rejet de la maternité : d'abord, à travers le rejet de la figure maternelle, puis à travers une écriture prenant la forme d'un manifeste pour la non-maternité. Nous explorerons tout d'abord les images de filiation tourmentée dans ces textes, puis étudierons la figure de la non-maternité volontaire comme rejet paradoxal de la mémoire, de la post-mémoire et du poids du passé et d'une certaine forme de féminité, afin de mettre en lumière la façon dont ces écrivaines contribuent à une redéfinition du concept de maternité en justifiant le choix de leurs narratrices de ne pas se reproduire, ce qui leur permet de ré-engen(d)rer la mémoire

${ }^{7}$ Lê et Sautière ont récemment été incluses dans une étude de Natalie EDwards (Voicing Voluntary Childlessness: Narratives of Non-Mothering in French, Oxford, Peter Lang, 2016) ; cependant, pas au prisme du genre et de la (post-)mémoire.

${ }^{8}$ M. Hirsch et N. K. Miller (eds.), Rites of Return: Diaspora Poetics and the Politics of Memory, New York, Columbia University Press, 2011.

9 L. LÊ, « J'aime que les livres soient des brasiers », Télérama, 20 août 2010, http:// www.telerama.fr/livre/linda-le-j-aime-que-les-livres-soient-des-brasiers,59204.php (consulté le 26 juillet 2018). 
à travers cette non-maternité, remettant ainsi en question les notions idéalisées de racines ${ }^{10}$ et visant à définir une nouvelle norme. Nous démontrerons que les trois textes inclus dans cette étude peuvent également être interprétés comme des tentatives de se réapproprier son histoire et de dépasser le statut victimaire.

Bien que traitant de traumatismes personnels et historiques différents, ces trois récits se prêtent tout particulièrement à une lecture dialogique, dans la mesure où tous trois s'articulent autour de notions similaires. Ces écrivaines se rejoignent également dans leur utilisation de la figure de la mère monstrueuse, voire métaphoriquement infanticide, afin de se réapproprier leur histoire dont elles ont le sentiment qu'elle a été étouffée par les discours masculins dominants (Loridan-Ivens et Lê) et de donner une voix à celles qui n'en ont pas (Guérin). La figure de l'infanticide, allant à l'encontre du discours habituel idéalisé tenu dans les récits masculins sur la maternité comme ultime vestige d'humanité dans des situations de déshumanisation extrême, fournit à ces femmes écrivains l'occasion d'un discours genré soulignant la subjectivité sexuée de leur expérience mémorielle particulière. De manière plus modérée car moins littérale, la problématique de l'infanticide métaphorique constitue également un outil essentiel dans les revendications des trois auteures incluses dans cette étude. Chez Lê, la narratrice doit tuer symboliquement l'enfant qu'elle aurait pu avoir afin de se donner naissance ; chez Loridan-Ivens, l'infanticide contient un double sens métaphorique : le sentiment de la narratrice d'avoir, à quatorze ans, été tuée à Auschwitz et de n'être depuis plus qu'une survivante et celui de choisir de ne pas se reproduire afin de rompre le cercle vicieux de la mémoire ; chez Guérin, le sacrifice, par le personnage de Maud, de l'enfant qu'elle aurait pu avoir lui permet de s'émanciper du poids de la maltraitance psychologique maternelle, évitant ainsi de perpétuer une reproduction mortifère. Nous soulignerons notamment l'omniprésence de la figure de la mère infanticide et du brouillage des catégories sexuelles et généalogiques dans ces récits.

\section{Linda Lê : (re)naître grâce à l'écriture de la non-maternité}

Linda Lê est née en 1963 à Dalat (Viêtnam). En 1969, sa famille déménage à Saïgon pour échapper à la guerre. Puis, en 1977, elle part vivre en France avec sa mère et ses trois sœurs. Dans son court texte épistolaire intitulé A l'enfant que je n'aurai pas, Lê s'adresse à son fils imaginaire en ces termes : « Tu m'as aidée à me transcender ${ }^{11}$. Ainsi, les rôles traditionnels sont inversés et en étant, dans un sens, sacrifié, l'enfant qu'elle n'a pas eu lui a permis de renaître. Là se trouve l'une des différences principales entre les autres textes de cette étude et celui de Lê : en effet, A l'enfant que je n'aurai pas est centré sur ce " toi », l'enfant qui ne naîtra jamais, qui se trouve ainsi placé dans une position sacrificielle permettant la catharsis de sa mère. D'une certaine façon, la lettre de Lê se présente comme une forme métaphorique d'infanticide, dans la mesure où, en sacrifiant son enfant non-né dont elle a déjà imaginé la vie tout entière dans les moindres détails, elle peut se libérer et se transcender. Elle précise même : «Ma fascination allait plutôt à Électre, la fille

${ }^{10}$ Contrairement à plusieurs études récentes, dont celle de M. Hirsch et N. K. MiLler, op. cit.

11 L. LÊ, A l'enfant que je n'aurai pas, op. cit., p. 63. 
vengeresse, et à Médée, la mère infanticide $»^{12}$. Le mot sur le papier donne chair à l'enfant et, tandis que Lê prétend que : « Je serai toujours la fille, libre d'entraves, et non la mère, aux multiples obligations. [...] Un individu qui se respecte ne se glorifie ni de son ascendance ni de sa descendance ${ }^{13}{ }^{13}$, cette lettre ouverte à l'enfant qu'elle n'a pas eu officialise sa non-maternité tout en la positionnant paradoxalement comme mère, en dépit de son désir de demeurer éternellement en position de fille.

A l'enfant que je n'aurai pas revêt donc la forme d'une lettre adressée par la narratrice à « toi, l'enfant que je n'aurai pas » ${ }^{14}$ et se lit comme un manifeste pour la non-maternité dans lequel la narratrice expose en détail les arguments sous-tendant sa décision de ne pas se reproduire. Cependant, à la lecture de la lettre, il devient rapidement évident que l'autre thème central se dessinant en filigrane est celui de la relation traumatisante et abusive qu'a connue Lê avec sa mère, qu'elle et ses sœurs surnommaient "Big Mother $\|^{15}$, ainsi que la transposition et l'anachronisme des traditions et de la culture vietnamiennes en France. Elle se souvient avec précision de son écrasant sentiment d'oppression par les attentes élevées de sa mère quant à ce que la « féminité » se devait d'être, quand elle n'était pas victime de violences physiques et psychologiques ouvertes. Le traumatisme et la terreur étaient tels qu'elle écrit : « C'est seulement aux alentours de la trentaine que j'ai pu vaincre quelque peu ma terreur d'elle ${ }^{16}$. En évoquant ses années d'adolescence, elle écrit :

Déjà, à l'époque, je me jurais de ne jamais être mère, pour ne pas donner à mes enfants l'éducation que j'avais reçue. Mes sœurs pensaient pouvoir conjurer la fatalité de la répétition, moi, à l'inverse, je craignais de reproduire des mécanismes d'autoritarisme ${ }^{17}$.

Ainsi, bien que l'ouvrage de Lê soit dédié à cet enfant non-né auquel elle s'adresse, la figure de l'enfant imaginaire est éclipsée par celle de la mère tout puissante, incarnation de traditions et d'un héritage culturel vietnamien perçus comme rigide, à la manière d'un carcan, ce qui n'est pas sans évoquer le roman autofictionnel de Marguerite Duras, $L^{\prime}$ Amant ${ }^{18}$, où la figure de l'amant se trouve en vérité supplantée par celle de la mère et par cette relation mère-fille tourmentée qui constitue le véritable sujet du texte. Cette problématique fait également écho au texte de Guérin.

Cependant Lê, tout comme les autres auteures de cette étude, ne manque pas de souligner la difficulté pour une femme à se positionner dans une société qui accorde une telle valeur à la maternité et qui exerce des pressions constantes sur les femmes pour qu'elles se reproduisent. Ainsi Lê évoque-t-elle en ces termes sa non-maternité : 《 mon insoumission à la loi de la reproduction ${ }^{19}$, faisant de la non-maternité un acte politique contestataire, et établit un parallèle entre l'athéisme et la non-maternité,
${ }^{12}$ Ibid., p. 33.
${ }^{13}$ Ibid., p. 10.
${ }^{14}$ Ibid., p. 7.
${ }^{15}$ En anglais dans le texte. Ibid., p. 12.
16 Ibid., p. 29.
${ }^{17}$ Ibid., p. 18.
${ }_{18}$ M. Duras, L'Amant, Paris, Éditions de Minuit, 1984.
19 L. LÊ, A l'enfant que je n'aurai pas, op. cit., p. 34. 
dans une société où la maternité est érigée au rang de dogme religieux ${ }^{20}$ : « Ni mère ni épouse, je m'échinais à être une trouveuse de la littérature sans adhérer à aucune chapelle ${ }^{21}$, « [sans] porter un embryon dans [ma] matrice ${ }^{22}$. Après sa tentative de suicide, et le séjour à Sainte Anne qui lui fait suite, elle s'entend dire par des psychiatres : « Mon caractère se bonifierait dès lors que je serais mère, je n'aurais plus le cafard $»{ }^{23}$. Semblant faire écho à l'ouvrage séminal de la féministe française Béatrice Didier, L'Écriture-femme ${ }^{24}$, sur la relation entre création littéraire et enfantement, tout en le remettant en question et le parodiant, Lê déclare que « la mise au jour d'une fiction n'équivaut pas à l'éclosion en soi d'un germe de vie $»^{25}$. Tandis que, pour Didier, la création littéraire est équivalente à la procréation intellectuelle, ce n'est pas le cas pour Lê, ce qui ajoute un surcroît de complexité à sa non-maternité volontaire. D'ailleurs, chez Lê, la création littéraire et la procréation sont présentées comme s'excluant mutuellement, l'une ne se sublimant pas en l'autre : «J'appréhendais que ma veine romanesque ne se tarisse si je me dévouais à mes poupons $»^{26}$. Le double sens du terme " poupon », désignant à la fois un nouveau-né et une poupée, souligne le caractère finalement non-réel et abstrait de la maternité pour l'auteure.

Lorsqu'elle ressent les premiers symptômes d'un trouble psychiatrique, elle se trouve en proie à des hallucinations dans lesquelles « la Seine charriait des fœtus expulsés avant terme ${ }^{27}$, ce qui souligne le motif de l'infanticide métaphorique à travers le prisme duquel nous appréhendons ce texte, le récit épistolaire pouvant se concevoir comme le tombeau de papier symbolique réceptacle du deuil de la maternité par la narratrice qui se trouve au tournant biologique de sa vie que constitue la ménopause. L'image des fœtus avortés charriés par la Seine évoque, à notre sens, les textes d'Emma Santos ${ }^{28}$, qui place la maternité délirante, les fœetus avortés et ses fantasmes d'autoreproduction au cœur de ses récits autobiographiques de l'expérience

${ }^{20}$ Il convient ici de rappeler la devise du gouvernement de Vichy, « Travail, famille, patrie ", ayant trouvé ses racines dans un discours prononcé par Sadi Carnot dès 1842, et dont l'influence perdure malgré sa disparition officielle, notamment dans la Fête des mères, la Caisse d'allocations familiales, et dont on a assisté à la résurgence lors des manifestations de contestation ayant secoué la France depuis 2013 à la suite de la remise en question de l'interdiction de la PMA aux femmes célibataires ou lesbiennes par le conseil de bioéthique. Les exemples normatifs rappelant la femme française au déterminisme de son rôle reproductif abondent dans le discours public contemporain, notamment dans la rhétorique entourant ces manifestations.

${ }^{21}$ L. Lê, A l'enfant que je n'aurai pas, op. cit., p. 36.

22 Ibid., p. 37.

${ }^{23}$ Ibid., p. 50.

24 B. Didier, L'Écriture-femme, Paris, Presses universitaires de France, 2004.

${ }^{25}$ L. LÊ, A l'enfant que je n'aurai pas, op. cit., p. 45.

${ }^{26}$ Ibid., p. 42.

27 Ibid., p. 35.

${ }^{28}$ Les textes autobiographiques d'Emma Santos s'articulent tous autour d'images de maternité délirante et de fœtus conquérant Paris, dans la mesure où elle attribuait ses symptômes psychiatriques à ses avortements forcés et répétés imposés par le corps médical patriarcal de la France des années 1970. Voir, entre autres, E. Santos, L'Itinéraire psychiatrique, Paris, des femmes, 1977, et La Malcastrée, Paris, des femmes, 1976. 
psychiatrique. Ainsi, Lê se replace implicitement dans la lignée féministe des années 1970 et du Mouvement de libération des femmes.

Par ailleurs, lors d'un entretien avec Catherine Argand pour L'Express, Lê déclare : « J'ai l'impression de porter en moi un corps mort. C'est sûrement le Vietnam que je porte comme un enfant mort $\gg{ }^{29}$. Ces propos évoquent ainsi un autre traumatisme lié au contexte historique, au-delà du contexte familial, replaçant la non-maternité choisie de Lê dans un désir métaphorique de couper les liens avec ses racines - si souvent idéalisées dans la littérature de l'exil - afin de surmonter le traumatisme de l'immigration. Enfin, d'un point de vue genré, il convient de remarquer que l'enfant que l'auteure aurait pu avoir ne peut se concevoir que comme de sexe masculin, ce qui s'inscrit dans la lignée logique du dégoût développé par la narratrice pour son propre corps, conséquemment à la relation mère-fille traumatisante qu'elle a subie durant son enfance et son adolescence.

\section{Maternité de Françoise Guérin : la non-maternité comme rupture du cycle mortifère et refus de transmettre}

La psychanalyste anglaise Jacqueline Rose dénonce, dans Mothers: An Essay on Love and Cruelty, les conséquences psychologiques destructrices du discours social entourant la maternité dans les sociétés occidentales contemporaines. Pour elle, la dépression du post-partum est en très grande partie causée par ce discours normatif et prescriptif selon lequel la maternité constitue l'épanouissement suprême vers lequel toute femme devrait tendre, ce qui exclut également la femme sans enfant de la communauté féminine, quelles que soient les raisons de sa non-maternité.

Maternité (2018), de Françoise Guérin, en dépit de son titre faussement simple, est un roman de 465 pages raconté à la deuxième personne du singulier et détaillant l'absence d'instinct maternel chez une jeune femme qui sombre peu à peu dans la dépression du post-partum la plus profonde. Le récit suit la protagoniste principale, Clara, de la conception de sa fille jusqu'aux neuf mois de celle-ci. Guérin s'inspire de son expérience clinique de psychothérapeute spécialiste des relations mère-enfant. Lors de la parution du roman, les critiques ont salué Maternité comme «bris[ant] le dernier tabou : la maternité comme accomplissement de la féminité ${ }^{30}$ - tabou ayant encore la vie dure, en France tout particulièrement. Ainsi, le récit de Guérin offre au lecteur une critique acerbe du mythe de l'instinct maternel et du discours médical et social opprimant qui l'accompagne. Faisant écho au texte de Lê, Guérin dresse un portrait satirique de la maternité comme nouvelle religion totalitaire et nous fournit une illustration sans concession des dégâts causés par l'injonction sociale également dénoncée par Jacqueline Rose, selon laquelle une mère ne doit vivre que pour son enfant, une mère est une mère et rien d'autre [" a mother must live only for her child, a mother is a mother and nothing else ${ }^{31}$ ].

${ }^{29}$ L. LÊ, Entretien avec Catherine Argand, « Lire », L'Express, $1^{\text {er }}$ avril 1999, https://www. lexpress.fr/culture/livre/linda-le_803102.html, (consulté le 10 janvier 2019). 2019).

${ }^{30} \mathrm{https} / / / \mathrm{www}$. albin-michel.fr/ouvrages/maternite-9782226400376), (consulté le 11 mars

${ }^{31}$ J. Rose, Mothers: An Essay on Love and Cruelty, New-York, Farrar, Straus \& Giroux, 2018, p. 78. 
La narration à la deuxième personne du singulier, dans un style concis et sans fioritures, loin de créer l'espace dialogique escompté, engendre plutôt un sentiment dérangeant d'étouffement et d'oppression, contribuant à l'objectification de Clara en tant que mère, comme si elle ne pouvait plus prétendre à la moindre intériorité. La confrontation de Clara à l'absence d'instinct maternel est notamment décrite en ces termes : «Tu as senti ta poitrine se gonfler de lait mais tu attends toujours la montée du désir, celui qui te fera consentir à ce qui n'est régi par aucun instinct ${ }^{32}$. Le fait que le nouveau-né n'est pas nommé durant les trois-cents premières pages de sa vie renforce l'expression incisive du tourbillon de la dépression dans laquelle s'enfonce peu à peu Clara, au point d'en venir à désirer commettre un infanticide.

Au fil du récit, il apparaît que le mal-être de Clara est lié à son enfance traumatisante, sous l'emprise d'une mère toxique et perverse narcissique, que la naissance de sa fille ravive. Pour Rose, la dépression post-natale est étroitement liée au silence imposé à la voix maternelle - « Perhaps most important of all, if you ignore the more disturbing narratives that are there to be read $\gg{ }^{33}$ - ainsi qu'aux injonctions sociales et familiales plaçant la jeune mère en proie à la projection de tous les maux sur sa personne "What on earth do we expect, as long as society continues to believe it has the right to trample over the mental lives of mothers? $\gg{ }^{34}$. Le récit de Guérin présente d'ailleurs explicitement la décision de Clara de devenir mère comme l'intériorisation d'une injonction extérieure, et non le fruit d'un désir personnel : « Déjà que, nullipare persévérante, tu te sens constamment écrasée par leur multiparité triomphante $! \gg{ }^{35}$.

J'aimerais m'attacher plus particulièrement au personnage de Maud, la sœur aînée de Clara, qui est d'abord présentée comme étant affligée par une stérilité d'origine inexpliquée ${ }^{36}$, ce qui l'a conduite, avec son mari, à adopter deux jeunes orphelins africains. Au fil du récit, il s'avère cependant que la non-maternité de Maud relève en fait d'un choix conscient de ne pas se reproduire, par peur de perpétuer malgré elle le schéma maternel destructif. Ainsi, Guérin présente au lecteur la trajectoire parallèle de deux sœurs dont la relation mère-fille destructrice a influencé leur propre ressenti vis-à-vis de la maternité et les a empêchées de ressentir ce potentiel «instinct » de reproduction, qui n'a justement pas pu se construire par l'intériorisation de l'éducation. Tandis que Clara se réfugie derrière la distance d'un champ sémantique purement médical, annonçant, par exemple, qu'elle est « primigeste » ${ }^{37}$, espérant ainsi nier l'existence de ce fœetus dont elle sent qu'elle devrait pourtant se réjouir ( « Ne rien dire. Ne pas risquer, par la parole, de donner consistance à la chose ${ }^{38}$ ), Maud choisit de passer par « [t]ant de douloureux renoncements pour que l'histoire familiale n'entache pas sa vie de femme ${ }^{39}$. Partie vivre à Sydney, en Australie, dans l'espoir que l'éloignement géographique aux antipodes de la France suffise à instaurer

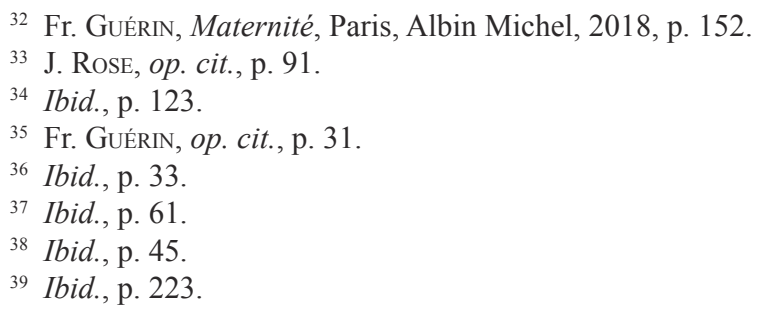


l'éloignement psychologique de sa mère tentaculaire, hypocondriaque et égocentrique, elle est pourtant contrainte au douloureux constat selon lequel « l'enfance est inscrite dans ta chair $\gg{ }^{40}$ et, lors d'une conversation avec Clara, elle est forcée de reconnaître que la transmission du traumatisme passe outre les contraintes génétiques :

S'installer au bout du monde ne suffisait pas. Maud a cru, en adoptant, se libérer du poids des générations. Au diable les ancêtres au sang corrompu. Sa chair ne donnerait pas le jour à leur descendance. Il fallait que quelqu'un prenne la décision radicale d'interrompre la lignée, quitte à faire taire ses entrailles torturées par le désir. Tirer un trait sur l'innommable.

C'était la seule solution.

[...] malgré son sacrifice l'innommable était bien là, dans la rencontre avec ces petits êtres tombés d'un autre corps que le sien. La transmission n'avait que faire de la génétique, elle qui, pour s'accomplir, possédait déjà le regard de Maud, ses bras, ses mots et jusqu'à son silence ${ }^{41}$.

Vers la fin du récit, le lecteur découvre que le comportement obsessionnel de la mère concernant l'hygiène, féminine en particulier, et sa conviction soudaine que le bébé de Clara se laisse mourir de faim car elle est atteinte de syphilis, et non pas parce qu'elle souffre d'anorexie du nourrisson causée par le manque d'attachement de sa mère, s'expliquent par la transmission transgénérationnelle d'un traumatisme lié à la Seconde Guerre mondiale et à la syphilis, maladie honteuse par excellence, cristallisé par la mère sur ses deux filles, dont la féminité est considérée d'emblée comme une souillure. Ainsi, dans cette relation mère-filles destructrice, les obsessions corporelles et la dictature de la propreté en sont venues à remplacer toute autre forme de communication, si bien que les deux sœurs constatent que «Porte close, c'est ce qui définit le mieux les relations au sein de ta famille $\gg{ }^{42}$, faisant ici écho à l'essai de Marianne Hirsch, Waiting behind closed doors, sur la relation mère-fille décrite par Duras dans son ouvrage autofictionnel L'Amant.

Dans cette famille lestée du poids du traumatisme transgénérationnel, la construction sociale et familiale de la maternité comme instinct n'a pu avoir lieu et a été remplacée par une perception de l'enfant à venir comme être colonisateur et comme incarnation du passé plutôt que de l'avenir. Ainsi, enceinte, Clara s'interroge au sujet de sa mère : "Parfois, tu te demandes si elle a ressenti la même chose, lorsqu'elle t'attendait. Si tu as été, pour elle, une anguille ou quelque autre bestiole inquiétante dans ses desseins de colonisation... ${ }^{43}$. Les références symboliques liées à la formation psychanalytique de Guérin abondent dans le récit, à commencer par le nom de famille de Clara, qui est ironiquement « Verrier », dénotant à la fois l'absence d'attachement et la fragilité de son personnage. S'étant construites au sein de ce foyer où régnait la maltraitance psychologique, les deux sœurs se tiennent « depuis toujours au bord de la vie ${ }^{44}$. Il est donc présenté comme une conséquence logique que la maternité, étant ce qui ramène la femme à la chair, au corps et, donc, au cœur de la

\footnotetext{
${ }^{40}$ Ibid., p. 451.

${ }^{41}$ Ibid., p. 225.

${ }^{42}$ Ibid., p. 65.

${ }^{43}$ Ibid., p. 80.

${ }^{44}$ Ibid., p. 97.
} 
vie, devienne le lieu de cristallisation de l'état de stress post-traumatique des deux sœurs. D'ailleurs, lorsque Clara entreprend enfin de consulter une psychothérapeute pour l'aider à surmonter sa dépression et son rejet de sa fille, le lapsus de sa première phrase s'avère très révélateur : « je n'arrive pas à mourir mon bébé » ${ }^{45}$, révélant son désir d'infanticide refoulé.

\section{Marceline Loridan-Ivens : la non-maternité comme ultime libération de l'univers concentrationnaire}

Dans son ouvrage, Rose dénonce l'opprobre dont sont victimes les mères dans la société occidentale contemporaine, surtout les mères célibataires, mais aussi, à l'autre extrémité de la biologisation de l'existence féminine, la femme sans enfant. Cependant, Rose rappelle au lecteur la toute-puissance subversive de la non-maternité qui permet à la femme choisissant de ne pas se reproduire de mettre, en théorie, un terme à l'espèce humaine ${ }^{46}$. Cet aspect de la non-maternité choisie a pu ainsi apparaître à certains critiques comme particulièrement controversé dans le cas de Marceline Loridan-Ivens, survivante de la Shoah, c'est-à-dire d'un génocide ayant visé à l'éradication complète de la population juive d'Europe.

Tout d'abord, comme de nombreux survivants, il me semble que Marceline se décrit comme étant demeurée à jamais fixée dans l'atemporalité du traumatisme, ce qui, par définition, l'empêche de désirer devenir mère, puisqu'elle se sent comme une éternelle survivante, dans un entre-deux psychologique, figée à l'âge qu'elle avait au moment de sa déportation (quatorze ans) : « Le camp a attaqué son corps à défaut de l'avoir brûlé $\gg{ }^{47}$ écrit-elle, choisissant de parler d'elle à la troisième personne pour souligner plus encore la disruption du traumatisme de la Shoah dans son identité et la continuité de son existence, et évoquant son corps de survivante comme étant celui d'une sous-vivante. Dans un entretien accordé à Florent Georgesco pour Le Monde des livres à l'occasion de la sortie de L'Amour après, Marceline souligne : « le rapport à mon corps a été totalement ravagé par les camps ${ }^{48}$. L'enjeu consiste donc bien en une réincarnation de son histoire par l'écriture.

Dans son ultime ouvrage autobiographique, Loridan-Ivens prend donc le parti d'aborder un sujet demeuré tabou dans les études françaises de la Shoah. Ainsi, elle évoque le caractère controversé de ses préoccupations avec le fait qu' « [à] ces amies revenues, j'ai vaguement tenté de poser quelques questions, sur leur corps, leur désir, leur intimité, sur l'amour après. Elles se sont toutes fermées $\gg{ }^{49}$. Pour Marceline, au contraire, une place prépondérante est accordée au corps, afin de réincarner son

45 Ibid., p. 355.

46 " But, as feminists have long pointed out, by refusing to be mothers, women have the power to bring the world to its end. » (J. Rose, op. cit., p. 48) [Mais, comme les féministes le soulignent depuis longtemps, en refusant de devenir mères les femmes ont le pouvoir de mettre un terme à l'univers].

${ }^{47}$ M. Loridan-Ivens, L'Amour après, Paris, Grasset, 2018, p. 27.

${ }^{48}$ M. Loridan-Ivens, Entretien avec Florent Georgesco, Le Monde des livres, 20 janvier 2018. https://www.lemonde.fr/livres/article/2018/01/20/marceline-loridan-ivens-le-rapport-amon-corps-a-ete-totalement-ravage-par-les-camps_5244478_3260.html.

${ }^{49}$ M. Loridan-Ivens, L'Amour après, op. cit., p. 152. 
histoire. Pour elle, le traumatisme de la Shoah a pour conséquence principale une désincarnation et une désexualisation, liées à la visée déshumanisante de l'entreprise concentrationnaire :

Je sortais d'un monde qui nous avait retiré notre nom, notre personne, alors sitôt revenue à la vie, sans que je puisse nommer et donc comprendre ce qui m'était arrivé, j'ai cherché instinctivement à retourner vers moi, ne laissant que peu d'espace aux autres en moi $^{50}$.

Elle évoque ainsi son errance à son retour d'internement à Auschwitz, BergenBelsen et Theresienstadt, cherchant par le biais de brèves rencontres amoureuses à tromper le désespoir et à surmonter le syndrome du stress post-traumatique dont on ne parlait pas encore à l'époque :

Qu'avais-je cherché à allumer dans le regard de ces inconnus, sinon la certitude d'être vivante, une image de moi qui ne serait pas celle que je portais dans ma tête, qui me rendrait plus séduisante à moi-même. [...] Elle avait déjà deux tentatives de suicide derrière elle ${ }^{51}$.

Ainsi, l'entreprise de désexualisation du génocide nazi des Juifs d'Europe, dont les femmes constituaient la cible privilégiée à cause de leurs capacités reproductrices, est décrite par la suspension des signes de féminité du corps de l'adolescente : «Mes règles ne sont plus venues. [...] Au camp, j'étais à l'écart, toute petite parmi des détenues qui me toisaient ou me maternaient ${ }^{52}$. Dans une perspective de portée universelle des témoignages de la Shoah, domaine largement dominé par le discours masculin ${ }^{53}$, le sujet de l'absence de menstruation dans les camps reste très rarement évoqué, à l'exception du récit de Ruth Klüger ${ }^{54}$ et du roman historique de Valentine Goby ${ }^{55}$, tous deux datant des années 2000. Ainsi, Marceline décrit la phobie des relations sexuelles avec laquelle elle dut lutter pendant des années, la nudité et la corporalité étant demeurées pour toujours associées à l'univers concentrationnaire, ayant perverti sa sexualité et son rapport au corps féminin de manière irréparable :

[...] bien au-delà de la crainte de la première fois, bien au-delà du risque de tomber enceinte, je fuyais mon propre corps, sa mise à nu, à jamais associée pour moi à l'ordre d'un nazi, à son regard humiliant tandis qu'on nous rasait la tête et le sexe, à son verdict : la mort ou le sursis. Jamais, avant le camp, je ne m'étais déshabillée devant quelqu'un, jamais je n'avais vu le corps de femmes nues, ni celui de ma mère, ni celui de mes sœurs. J'ai découvert le mien en même temps que je l'ai su condamné. [...] J'ai tout vu de la mort sans rien connaître de l'amour ${ }^{56}$.

\footnotetext{
${ }^{50}$ Ibid., p. 57.

${ }^{51}$ Ibid., p. 30.

${ }^{52}$ Ibid., p. 35. témoignages de femmes.

${ }^{54}$ R. KLÜGER, Refus de témoigner, Paris, Poche, 2005.

${ }_{55}$ V. Goby, Kinderzimmer, Arles, Actes Sud, 2013.

${ }^{56}$ M. Loridan-Ivens, L'Amour après, op. cit., p. 34.
}

${ }^{53}$ La base de données du Mémorial de la Shoah de Paris ne comporte que 5\% de 
Par conséquent, l'écrivaine explique qu' « elle garde un corps d'enfant » ${ }^{57}$, expression physique de l'atemporalité du traumatisme, et ses amants s'émerveillent de la cohabitation en elle de la maturité provoquée par l'internement et d'un comportement d'éternelle adolescente : «Quel âge as-tu, Marceline, en dehors de ces additions chronologiques qui te concernent si peu, puisque, à quatorze ans, tu as tout appris et qu'à trente-trois en toi rien n'a vieilli ? ${ }^{58}$.

Puis, évoquant son mariage sans amour à un jeune homme juif, Marceline ironise sur le dédoublement psychologique caractéristique des survivants ${ }^{59}$ : «Ainsi avançait la survivante, tandis que la jeune fille s'était mise en règle avec sa mère et la société ${ }^{60}$. Sa mère, avec laquelle les relations sont conflictuelles, cristallise ses inquiétudes sur la possibilité que sa fille ait pu être violée à Auschwitz, ce qui aurait compromis son mariage. Le mari de la narratrice est ainsi décrit en ces termes : « [...] Il avait, comme moi, mission fixée par des parents survivants de se marier vite, de faire des enfants et d'oublier $»^{61}$. Ici, l'enfant est donc censé représenter l'oubli et l'ouverture sur l'avenir. C'est précisément à ce discours prédominant sur la maternité cathartique que s'attaque Marceline, ayant à cœur de remettre en question l'injonction de reproduction comme condition sine qua non de retour à la norme et à la normalité. Lorsqu'elle revoit ses anciennes camarades de captivité, dont Simone Veil, elle ne peut s'empêcher de se demander si ces femmes faisant le choix de devenir mères pour oublier ne restent finalement pas prisonnières des injonctions sociales et souligne à plusieurs reprises que « [i]l n'y eut, après les camps, plus aucun donneur d'ordres dans ma vie ${ }^{62}$. Ainsi, elle décrit le vide ressenti par Ida, survivante ayant cru à la catharsis apportée par le mariage et la maternité et « qui découvre qu'une vie trop calme ne guérit de rien, bien au contraire. Elle se dédouble elle aussi $»{ }^{63}$.

À travers ce thème et ces rencontres avec d'anciennes détenues, c'est au procès de la société française de l'immédiat après-guerre que se livre Marceline, société qui remplace les barbelés d'Auschwitz par ceux de l'injonction à la reproduction, et dont il faut « fuir leurs pièges, leurs barbelés invisibles " ${ }^{64}$. Ainsi, la non-maternité de l'auteure, loin de l'exclure de la communauté des survivantes, la rapproche de ces femmes condamnées à la maternité, dans une même lutte :

Je ne peux m'empêcher de superposer nos corps, nos enfants, nés ou pas, nos histoires, comme une question qui nous était posée à toutes, puisque la société n'attendait qu'une seule chose de nous : mariez-vous et procréez. Qu'allions-nous devenir? Avions-nous le choix ? Moi je n'ai jamais voulu d'enfant, je ne suis jamais tombée enceinte, sans avoir fait attention, ni jamais pris la pilule. Je n'ai signé le
${ }^{57}$ Ibid., p. 37.
${ }^{58}$ Ibid., p. 36.
59 À ce sujet, voir notamment les textes de Charlotte Delbo, dont sa trilogie Auschwitz et après I, II et III (Paris, Éditions de Minuit, 1971).
${ }^{60}$ M. Loridan-Ivens, L'Amour après, op. cit., p. 54.
${ }^{61}$ Ibid., p. 38-39.
${ }^{62}$ Ibid., p. 58.
${ }^{63}$ Ibid., p. 69.
${ }^{64}$ Ibid., p. 71. 
manifeste des 343 salopes que par solidarité. Mon corps était sec. Rien n'a jamais germé en moi ${ }^{65}$.

Le récit trouve sa valeur unique et sa puissance dans cette dénonciation du carcan social de l'après-guerre s'avérant aussi destructeur que l'expérience concentrationnaire, où survivre signifie passer d'une prison à une autre :

[...] est-ce la fin de Caramel, jeune fille si drôle, si franche, cachée du monde, dévorée par l'enfant qu'elle porte ? Comme moi tantôt survivante, tantôt jeune fille. Tout le monde avançait masqué. Mais nous plus encore. Le monde ne nous autorisait rien ${ }^{66}$.

Ainsi Caramel se trouve-t-elle « dévorée par l'enfant qu'elle porte », inversion généalogique hautement symbolique.

Cependant, au fil du récit, après avoir été présentés comme source d'angoisse et de rejet social, la non-maternité et l'anticonformisme deviennent peu à peu libération : la libération des camps est, chez Loridan-Ivens, présentée comme indissociable de la libération de la femme. Sa survie et sa renaissance s'inscrivent dans le Mouvement de libération des femmes avant la lettre, comme elle a à cœur de le revendiquer tout au long du récit, citant notamment la communauté de femmes au sein de laquelle elle évolue à Saint-Germain-des-Prés.

Enfin, L'Amour après souligne également les autres voies et réseaux empruntés pour la transmission, qui se fait en-dehors de la reproduction. Tout d'abord, Marceline devient la mère de son mari, Joris Ivens, alors qu'il a l'âge d'être son père. Puis, elle choisit d'ouvrir son récit sur une conversation avec un jeune homme rencontré lors d'une soirée dansante alors qu'elle était déjà âgée de plus de 80 ans :

Est-ce que tu sais que des enfants ou des petits-enfants de déportés se font tatouer le numéro de leurs parents ? [...] Alors ce numéro, je te le donne. Je n'ai pas d'enfant. Je vais mourir bientôt, mais je ne veux pas que cette histoire meure avec moi. Prends ce numéro et note-le sur ton bras ${ }^{67}$.

Ainsi, la transmission passe toujours par le corps mais en contournant la biologie, puisque l'auteure demande à son jeune héritier d'inscrire à son tour son numéro de matricule de déportée sur son bras. Comme chez Guérin, pour contourner la transmission mortifère du traumatisme non-résolu, Loridan-Ivens a recours à l'adoption - même s'il ne s'agit pas d'une adoption administrative mais plutôt de la création d'une sororité :

Je me souviens aussi de Caramel, en fugue, recherchée par sa mère, qui proposa que je l'adopte, puisque je venais d'avoir vingt et un ans. D'une certaine manière nous nous étions adoptées, au moins momentanément, protégées peut-être, en tout cas, écoutées. [...] la plupart de mes amies revenues de Birkenau ont eu envie de calme, d'un toit, d'un mari et d'enfants qui définissent ce qu'elles feraient demain et après-demain. Pas moi ${ }^{68}$.
${ }^{65}$ Ibid., p. 67.
${ }^{66}$ Ibid., p. 63.
${ }^{67}$ Ibid., p. 12-13.
${ }^{68}$ Ibid., p. 59. 
Malgré le spectre de la Shoah, le récit autobiographique de Loridan-Ivens adopte un ton léger et optimiste, affirmant son désir d'échapper à la folie suicidaire qui gangrène sa famille et le fait qu'elle aime sa liberté plus que tout. Finalement, la nonmaternité permet, au soir de sa vie, de se ré-engen(d)rer par l'écriture et de surmonter le dédoublement et l'atemporalité du traumatisme génocidaire : « J'exhume enfin ma guerre. Je suis sur le point de rassembler la jeune fille et la survivante. De devenir une femme ${ }^{69}$.

\section{Conclusion}

Les trois récits inclus dans cette étude présentent différents argumentaires pour la non-maternité choisie dans un contexte intensément traumatique, sans pour autant se limiter à une présentation du choix de ne pas se reproduire comme un choix par défaut ou «non-choix ${ }^{70}$. Il s'agit plutôt de remettre en question le discours galvaudé concernant l'instinct maternel afin de démontrer comment un contexte mémoriel traumatisant empêche non seulement l'avènement de cet instinct maternel mythifié mais le remplace par l'instinct de non-maternité ou cette métaphore du « corps silencieux » à laquelle ont recours les femmes interviewées par Peterson et Engwall et sous-entendue par les trois auteures ci-incluses, par le biais de diverses métaphores. Le thème de la non-maternité volontaire, notamment à travers le deuil de l'enfant non-né et de l'infanticide textuel ou réel, permet à ces écrivaines de se réapproprier leur histoire au prisme du genre, afin de tenter de dépasser le poids du passé. Les textes de Lê, Guérin et Loridan-Ivens ont en commun de souligner le brouillage des frontières généalogiques et des catégories de genre causé par la Shoah, l'immigration, ou une figure maternelle toxique, afin d'exprimer le contexte traumatique dans lequel elles écrivent. Il convient de remarquer que la libération ultime de la femme figée dans sa place biologique de fille recevant passivement le poids de la mémoire transmise notamment chez Guérin et Lê - est paradoxalement décrite comme se produisant par le choix de demeurer sans enfant.

En 2009, Michael Rothberg ${ }^{71}$ dénonçait les problèmes éthiques soulevés par la figure de l'enfant placée au cœur des débats mémoriels actuels entourant des événements historiques catastrophiques, en analysant le film Caché, de Michael Haneke, dont l'intrigue est construite autour de la transmission transgénérationnelle du trauma de la Guerre d'Algérie et de la Shoah. Les textes de Lê, Loridan-Ivens et Guérin peuvent se lire comme le contrepied à ce problème. Le rejet de la maternité par leurs narratrices souligne un rejet de leur propre figure maternelle, ce qui, au fil du texte, s'apparente à une crise de la mémoire et des origines allant à l'encontre de la politique actuelle de la mémoire et de l'idéalisation des « racines » évoquée dans le collectif Rites of Return dirigé par Hirsch et Miller. Ainsi, le rejet de la maternité devient refus de transmettre et, de ce fait, incarne l'espoir d'une émancipation du poids de la mémoire. La revendication du choix de ne pas se reproduire devient une figure de style utilisée

${ }^{69}$ Ibid., p. 104.

${ }^{70}$ L. LANGER, "The Dilemma of Choice in the Deathcamps », Holocaust: Religious and Philosophical Implications, New-York, Paragon House, 1989, p. 222-232.

${ }^{71}$ M. Rothberg, Multidirectional Memory: Remembering the Holocaust in the Age of Decolonization, Stanford, Stanford University Press, 2009, p. 121. 
comme moyen de ré-engen(d)rer la mémoire à travers une crise de la maternité dans la littérature francophone contemporaine, où l'enfant devient métaphore de la mémoire et où, par conséquent, la (post-)mémoire pervertit les relations familiales au point que l'enfant n'est plus perçu comme représentant l'avenir et un nouveau commencement mais devient le reposoir d'un passé encombrant et un mémorial vivant à travers lequel la mère risque de perpétuer son propre traumatisme. Demeurer sans enfant vise ainsi à se libérer, non seulement des contraintes de la maternité mais également du poids du passé. Paradoxalement, ne pas se reproduire permet à ces auteures et leur narratrice de faire le deuil du passé et de surmonter les entraves de l'histoire familiale.

Cette posture permet également à ces écrivaines de remettre en question leur position de sujet réifié par les discours dominants sur la maternité, tout en se réappropriant leur histoire, afin de prétendre à une nouvelle place au sein du canon littéraire. Cette figure partagée de la non-maternité à laquelle elles ont recours peut se percevoir comme une nouvelle voix émergeante dans l'écriture féminine francophone contemporaine touchant à la subjectivité sexuée de l'écriture de soi et du traumatisme.

\section{Bibliographie}

Delbo C., Aucun de nous ne reviendra. Auschwitz et après I, Paris, Éditions de Minuit, 1970.

Delbo C., Une Connaissance inutile. Auschwitz et après II, Paris, Éditions de Minuit, 1970.

Delbo C., Mesure de nos jours. Auschwitz et après III, Paris, Éditions de Minuit, 1971.

Didier B., L'Écriture-femme, Paris, Presses universitaires de France, 2004.

Donath Or., « Regretting Motherhood. A Sociopolitical Analysis », Signs, 40, 2015, p. 343-367.

Duras M., L'Amant, Paris, Éditions de Minuit, 1984.

Edwards N., Voicing Voluntary Childlessness : Narratives of Non-Mothering in French, Oxford, Peter Lang, 2016.

Engwall, K. et Peterson H., "Silent Bodies: Childfree Women's Gendered and

Embodied Experiences », European Journal of Women's Studies, 20, 2013, p. 376-389.

Giongio A., Writing Mothers and Daughters : Renegotiating the Mother in Western European Narratives by Women, New York, Berghahn Books, 2002.

Goby V., Kinderzimmer, Arles, Actes Sud, 2013.

GuÉRIN Fr., Maternité, Paris, Albin Michel, 2018.

Hirsch M., The Mother/Daughter Plot. Narrative, Psychoanalysis, Feminism, Bloomington et Indianapolis, Indiana University Press, 1989.

Hirsch M., « Past Lives : Postmemories in Exile », Poetics Today, 17/4, 1996, p. 659686.

Hirsch M. et Miller N. K., Rites of Return : Diaspora Poetics and the Politics of Memory, New York, Columbia University Press, 2011. 
KLÜGER R., Refus de témoigner - une jeunesse, Paris, Poche, 2005.

LAnger L., "The Dilemma of Choice in the Deathcamps », Holocaust: Religious and Philosophical Implications, New-York, Paragon House, 1989, p. 222-232.

LÊ L., A l'Enfant que je n'aurai pas, Paris, NiL, 2011.

LÊ L., Entretien, Télérama, 20 août 2010. http://www.telerama.fr/livre/linda-le-jaime-que-les-livres-soient-des-brasiers,59204.php. Dernière consultation le 26 juillet 2018.

Letherby G., "Childless and bereft ? Stereotypes and realities in reaction to "voluntary" and "involuntary" childlessness and womanhood », Sociological Inquiry, 72/1, 2002, p. 7-20.

Loridan-Ivens M., L'Amour après, avec Judith Perrignon, Paris, Grasset, 2018.

Loridan-Ivens M., Entretien avec Florent Georgesco, Le Monde des livres, 20 janvier 2018. https://www.lemonde.fr/livres/article/2018/01/20/marceline-loridan-ivensle-rapport-a-mon-corps-a-ete-totalement-ravage-par-les-camps_5244478_3260. html. Consulté le 22 mars 2019.

Mokeddem M., Je dois tout à ton oubli, Paris, Grasset, 2008.

Moore J., « Reconsidering Childfreedom: A Feminist Exploration of Discursive Identity Constructions in Childfree LiveJournal Communities », Women's Studies in Communication, 37/2, 2014, p. 159-180.

Morell C., Unwomanly Conduct : The Challenges of Intentional Childlessness, New York, Routledge, 1994.

Morison T., MACLEOD C., et al., « Stigma Resistance in Online Childfree Communities: The Limitations of Choice Rhetoric ", Psychology of Women Quarterly, 40/2, 2016, 184-198.

Peterson H., « Fifty Shades of Freedom. Voluntary Childlessness as Women's Ultimate Liberation », Women's Studies International Forum, 53, 2015, p. 182-191.

Rich Ad., Of Woman Born: Motherhood as Experience and Institution, New York, Norton, 1976.

Rose J., Mothers: An Essay on Love and Cruelty, New-York, Farrar, Straus \& Giroux, 2018.

Rothberg M., Multidirectional Memory: Remembering the Holocaust in the Age of Decolonization, Stanford, Stanford University Press, 2009.

SANTOS E., Effraction au réel, Paris, des femmes, 2006.

Santos E., L'Itinéraire psychiatrique, Paris, des femmes, 1977.

SAntos E., La Malcastrée, Paris, des femmes, 1976.

SAutière J., Nullipare, Paris, Gallimard, 2008.

Wajsbrot C., L'Hydre de Lerne, Paris, Zulma, 2011.

Wajsbrot C., Mémorial, Paris, Zulma, 2005.

Waxman Z., Women in the Holocaust, Oxford, Oxford University Press, 2017. 